\title{
Atividades antimicrobiana e leishmanicida das sementes de Pterodon emarginatus Vogel
}

\author{
Rafael C. Dutra, ${ }^{1}$ Fernanda G. Braga, ${ }^{2}$ Elaine S. Coimbra, ${ }^{2}$ Adilson D. Silva, ${ }^{3}$ \\ Nádia R. Barbosa ${ }^{*, 1}$ \\ ${ }^{1}$ Departamento de Alimentos e Toxicologia, Faculdade de Farmácia e Bioquímica, Universidade Federal de Juiz de \\ Fora, Martelos, 36036-330 Juiz de Fora-MG, Brasil, \\ ${ }^{2}$ Departamento de Parasitologia, Microbiologia e Imunologia, Instituto de Ciências Biológicas, Universidade \\ Federal de Juiz de Fora, Martelos, 36036-330 Juiz de Fora-MG, Brasil, \\ ${ }^{3}$ Departamento de Química, Instituto de Ciências Exatas, Universidade Federal de Juiz de Fora, Martelos, \\ 36036-330 Juiz de Fora-MG, Brasil
}

\begin{abstract}
RESUMO: O presente trabalho investigou as atividades antimicrobiana e leishmanicida das sementes de Pterodon emarginatus Vogel. O potencial antimicrobiano do óleo essencial (OE) obtido das sementes foi avaliado através dos testes de difusão em ágar (10, 25 e $50 \mathrm{mg}$ ) e determinação da concentração inibitória mínima e utilizou os microorganismos-padrão: Staphylococcus aureus ATCC 25923, Streptococcus mutans ATCC 25175, Pseudomonas aeruginosa ATCC 90271, Escherichia coli ATCC 10530 e Candida albicans ATCC 10231. A atividade leishmanicida do $\mathrm{OE}$ e frações $(6,25-100 \mu \mathrm{g} / \mathrm{mL})$ obtidas das sementes de P. emarginatus foram testadas, in vitro, sobre formas promastigotas de L. amazonensis e L. chagasi. O OE das sementes de P. emarginatus inibiu o crescimento somente de $S$. aureus $(\mathrm{CIM}=2,5 \mathrm{mg} / \mathrm{mL})$. As frações hexânica $\left(\mathrm{IC}_{50}=50,06\right.$ $\mu \mathrm{g} / \mathrm{mL})$ e butanólica $\left(\mathrm{IC}_{50}=46,65 \mu \mathrm{g} / \mathrm{mL}\right)$ apresentaram atividade frente às formas promastigotas de L. amazonensis, porém não apresentaram atividade frente a L. chagasi. Os resultados indicam que as moléculas bioativas presentes nas sementes de $P$. emarginatus podem ser utilizadas como protótipos para o desenvolvimento de fármacos e/ou como fonte de matérias-primas farmacêuticas.
\end{abstract}

Unitermos: Pterodon emarginatus, Leguminosae, atividade antibacteriana, leishmanicida.

\begin{abstract}
Antimicrobial and leishmanicidal activities of seeds of Pterodon emarginatus". The present work investigated the antimicrobial and leishmanicidal activities of seeds of Pterodon emarginatus. The tests of diffusion in agar $(10,25$ and $50 \mathrm{mg})$ and determination of minimum inhibitory concentration (MIC) were performed using essential oil (EO) obtained from seeds using the standard microorganisms: Staphylococcus aureus ATCC 25923, Streptococcus mutans ATCC 25175, Pseudomonas aeruginosa ATCC 90271, Escherichia coli ATCC 10530 and Candida albicans ATCC 10231. Leishmanicidal activity of the EO and fractions $(6.25-100 \mu \mathrm{g} / \mathrm{ml})$ obtained of seeds of $P$. emarginatus was evaluated in vitro using $L$. amazonensis and L. chagasi promastigote forms. The EO inhibited the growth of $S$. aureus $(\mathrm{MIC}=2.5 \mathrm{mg} / \mathrm{ml})$. The hexane $\left(\mathrm{IC}_{50}=50.06 \mu \mathrm{g} /\right.$ $\mathrm{ml})$ and butanol $\left(\mathrm{IC}_{50}=46.65 \mu \mathrm{g} / \mathrm{ml}\right)$ fractions showed activity against $L$. amazonensis promastigote forms, but did not against $L$. chagasi promastigote forms. The results indicate that the bioactive molecules present in the seeds of $P$. emarginatus can be used as prototype for the development of drug and/or as source pharmaceutical material.
\end{abstract}

Keywords: Pterodon emarginatus, Leguminosae, antibacterial activity, leishmanicidal.

\section{INTRODUÇÃO}

Um dos maiores problemas de Saúde Pública enfrentados nas últimas décadas foi o agravamento da resistência a antimicrobianos em cepas bacterianas, principalmente de origem hospitalar (Oliveira et al., 2006a,b; Coutinho et al., 2008). Atualmente, registra-se um aumento significativo na freqüência do isolamento de bactérias que eram reconhecidamente sensíveis aos fármacos de rotina usados na clínica, mas que se apresentam agora resistentes a quase todos os fármacos disponíveis no mercado, como ocorre com várias bactérias multirresistentes (Shiomori et al., 2002; Sakagami \& Kajamura, 2006).

Neste contexto, as plantas representam fontes valiosas de produtos para a manutenção da saúde humana, sendo seu uso mais difundido especialmente nos últimos anos, após numerosos estudos com produtos terapêuticos oriundos de plantas medicinais. Segundo a Organização Mundial da Saúde (OMS), as plantas medicinais são as melhores fontes para se obter uma variedade de medicamentos, e cerca de 
$80 \%$ da população mundial usa a medicina tradicional na busca de alívio de alguma sintomatologia dolorosa ou desagradável (Nascimento et al., 2000; Caetano et al., 2002; Corrêa et al., 2008; Sousa et al., 2008). A seleção de plantas, a partir de informações da medicina tradicional ou popular, pode conduzir a descoberta de moléculas promissoras com ação terapêutica (Agra et al., 2007).

As leishmanioses são doenças endêmicas causadas por protozoários do gênero Leishmania, ocorrendo em várias partes do mundo sendo responsáveis por elevados índices de morbidade e mortalidade. Essa zoonose compreende formas clínicas variadas e pode, classicamente, ser dividida em dois grupos principais: Leishmaniose Tegumentar Americana (LTA) e Leishmaniose Visceral (LV). As manifestações clínicas são resultantes de complexas interações entre o parasito e a resposta imune do indivíduo infectado (Grevelink \& Lerner, 1996; Herwaldt, 1999). No Brasil, a LTA e a LV ocorrem em todo o território nacional e são endêmicas nas regiões Norte e Nordeste, devido principalmente às características econômicas e culturais dessas populações, predominando nos estados da Bahia, Ceará, Piauí e Maranhão (Ministério da Saúde, 2000; Rath et al., 2003).

$\mathrm{O}$ tratamento das leishmanioses é feito à base de antimoniais pentavalentes $\left(\mathrm{Sb}^{+5}\right)$, anfotericina $\mathrm{B}$ e pentamidinas (isotionato e mesilato), quimioterápicos de elevado custo, usados por via parenteral e por prolongado período de administração, causando efeitos adversos, como: alterações cardíacas, renais, pancreáticas e hepáticas (Croft \& Yardley, 2002). Porém, desde a descoberta dos $\mathrm{Sb}^{+5}$, pouco tem sido feito para a descoberta de novos tipos estruturais para o tratamento dessa patologia. Entretanto, as dificuldades em relação à terapêutica atual têm conduzido a estudos com o objetivo de avaliar terapias alternativas, buscando, por exemplo, soluções na privilegiada biodiversidade brasileira, através da descoberta e desenvolvimento de novas moléculas com atividade terapêutica, ou no desenvolvimento de fitoterápicos nacionais para o tratamento das leishmanioses (Phillipson \& Wright, 1991a,b; Rocha et al., 2005).

Pterodon emarginatus Vogel (Leguminosae), conhecida popularmente como sucupira branca ou faveiro é uma árvore aromática nativa que mede de 5-10 metros de altura (Lorenzi \& Matos, 2002), facilmente encontrada na região do cerrado brasileiro e utilizada na medicina tradicional para o tratamento de reumatismo, dores de garganta, disfunções respiratórias (bronquites e amidalites), além das suas atividades antiinflamatória, analgésica, depurativa e tônica. As sementes são comercializadas em mercados populares por suas propriedades farmacológicas (Leite de Almeida \& Gottlieb, 1975; Arriaga et al., 2000; Agra et al., 2008).

Estudos fitoquímicos do gênero Pterodon têm demonstrado a presença de alcalóides nas cascas das árvores (Torrenegra et al., 1989), isoflavonas e alguns triterpenos (Marques et al., 1998) na madeira, e diterpenos (Fascio et al., 1976; Arriaga et al., 2000) e isoflavonas no óleo das sementes (Braz-Filho \& Gottlieb, 1971). O diterpeno 14,15-epoxigeranilgeraniol e alguns derivados, isolados de P. pubenscens têm sido associado com atividade protetora contra a penetração de cercárias de S. mansoni, parasito responsável pela esquistossomose, doença tipicamente tropical (Dos Santos Filho et al., 1972). Alguns destes constituintes químicos estão contidos em várias espécies vegetais com efeitos antibacterianos e leishmanicidas (Mathias \& Emily, 1993).

Baseado nos usos populares e nos constituintes químicos presentes nas sementes de $P$. emarginatus que sugerem seu potencial farmacológico, este estudo teve por objetivo avaliar as atividades antimicrobiana e leishmanicida das sementes de P. emarginatus Vogel.

\section{MATERIAL E MÉTODOS}

\section{Coleta do material vegetal}

Sementes da espécie $P$. emarginatus foram coletadas em setembro de 2006, no município de Três Marias/MG-Brasil e identificadas pela Dra. Fátima Regina Gonçalves Salimena do Departamento de Botânica, UFJF, Brasil. Uma exsicata da espécie foi depositada no Herbário CESJ da Universidade Federal de Juiz de Fora/MG-Brasil sob o número 48.077.

\section{Extração do óleo essencial}

O óleo essencial foi extraído em aparelho de Clevenger pelo método de hidrodestilação durante quatro horas. Foram pesados $30 \mathrm{~g}$ de sementes, as quais foram trituradas e adicionadas ao balão contendo $300 \mathrm{~mL}$ de água destilada. Após a extração, o óleo foi acondicionado em frasco de vidro âmbar e utilizado imediatamente para a realização das análises.

\section{Preparo das frações}

Para o preparo das frações, 30 gramas de sementes foram trituradas e submetidas à extração utilizando aparelho de Soxhlet. Após o acondicionamento das sementes ao cartucho e adaptação do balão de fundo redondo, a amostra foi submetida a extrações sucessivas com hexano, butanol e metanol até atingir o esgotamento do solvente. As frações hexânica (FH), butanólica (FB) e metanólica (FM) foram submetidas a rotaevaporação até eliminação completa do solvente. Tais frações foram utilizadas para avaliação da atividade leishmanicida.

\section{Atividade antimicrobiana}

A atividade antimicrobiana foi realizada 
utilizando os microorganismos-padrão: Staphylococcus aureus ATCC 25923, Streptococcus mutans ATCC 25175, Pseudomonas aeruginosa ATCC 90271, Escherichia coli ATCC10530 e Candida albicans ATCC 10231, obtidos da American Type of Culture Collection (ATCC). Para a realização desta atividade foram empregados o método de difusão em ágar (Mislivec et al., 1992; Swanson et al., 1992) e a determinação da concentração inibitória mínima (CIM) (Candan et al., 2003).

\section{Difusão em ágar}

A atividade antimicrobiana foi realizada através do método de difusão em ágar estabelecido por Mislivec et al. (1992) e Swanson et al. (1992). Os microorganismos foram cultivados em meio inclinado anti-1 (S. aureus, S. mutans e E. coli), Tryptone Soy Agar (TSA) (P. aeruginosa) e Sabouraud Dextrose Agar (SDA) (C. albicans) por um período mínimo de 24 horas a temperatura de $37^{\circ} \mathrm{C}$ em estufa bacteriológica. Após este período, uma suspensão microbiana foi padronizada utilizando solução fisiológica estéril $(\mathrm{NaCl}$ $9,0 \mathrm{~g} / \mathrm{L}$ ) contendo $10^{3}-10^{4} \mathrm{UFC} / \mathrm{mL}$. A suspensão foi diluída com solução fisiológica estéril até a obtenção de $25 \%$ de transmitância. As placas de Petri (dimensões: $15 \mathrm{~mm}$ de altura e $90 \mathrm{~mm}$ de diâmetro) foram preparadas com $22 \mathrm{~mL}$ de meio de cultura, resultando em um meio de cultura com $3 \mathrm{~mm}$ de profundidade. Cilindros (de aço inoxidável) estéreis (dimensões: $10 \mathrm{~mm}$ de altura e 6 $\mathrm{mm}$ de diâmetro) contendo 10,25 e $50 \mathrm{mg}$ do $\mathrm{OE}$ das sementes de $P$. emarginatus foram inseridos no meio de cultura. Todas as placas foram incubadas em ambiente aeróbico a temperatura de $37^{\circ} \mathrm{C}$ por 24 horas (bactérias) e temperatura de $22{ }^{\circ} \mathrm{C}$ por 48 horas (fungo). A inibição do crescimento foi medida, com auxílio de paquímetro, pela formação de halos, expressos em $\mathrm{mm}$. O ensaio foi realizado em triplicata e os resultados expressos como média \pm desvio padrão. Penicilina $(1 \mathrm{UI} / \mathrm{mL})$ e nistatina $(20 \mathrm{UI} / \mathrm{mL})$ foram utilizadas como fármacos de referência.

\section{Determinação da concentração inibitória mínima (CIM)}

Para a determinação da CIM, uma suspensão bacteriana (S. aureus ATCC 25923) foi preparada com solução fisiológica estéril $(\mathrm{NaCl} 9,0 \mathrm{~g} / \mathrm{L})$ a $25 \%$ de transmitância. A suspensão microbiana padronizada foi submetida à diluição seriada com solução fisiológica estéril e a contagem das unidades formadoras de colônias (UFC) foi realizada utilizando o ágar de contagem (PCA) após um período de 24 horas. O controle positivo foi preparado com $4 \mathrm{~mL}$ de caldo caseína inoculado com o S. aureus $\left(10^{3}-10^{4} \mathrm{UFC} / \mathrm{mL}\right)$ e $1 \mathrm{~mL}$ de solução fisiológica estéril. Para o controle negativo, foram adicionados $4 \mathrm{~mL}$ de caldo caseína estéril e $1 \mathrm{~mL}$ de solução fisiológica estéril. O procedimento foi realizado em triplicata. No preparo dos tubos-teste foram acrescentados $4 \mathrm{~mL}$ de caldo caseína inoculado e $1 \mathrm{~mL}$ de cada concentração da amostra-teste utilizada $(0,625$ - $10 \mathrm{mg} / \mathrm{mL}$ ). Os tubos foram incubados em ambiente aeróbico a temperatura de $37{ }^{\circ} \mathrm{C}$ por 24 horas. A CIM foi determinada através da observação de turvação dos meios de cultura, após o período de incubação (Candan et al., 2003).

\section{Atividade leishmanicida}

A atividade leishmanicida foi realizada de acordo com Braga et al. (2007). As formas promastigotas utilizadas para a realização do screening foram Leishmania amazonensis (MHOM/Br/75/Josefa isoladas de paciente com leishmaniose cutânea difusa) e Leishmania chagasi $(\mathrm{MHOM} / \mathrm{Br} / 74 / \mathrm{PP} 75$ isoladas de pacientes com leishmaniose visceral). As formas

Tabela 1. Atividade antimicrobiana do óleo essencial obtido das sementes de P. emarginatus contra microorganismos-padrão.

\begin{tabular}{|c|c|c|c|c|c|c|}
\hline \multirow[t]{3}{*}{ Microorganismos } & \multicolumn{3}{|c|}{ Zonas de inibição $(\mathrm{mm})^{\mathrm{a}}$} & & & \multirow{3}{*}{$\begin{array}{c}\text { CIM } \\
(\mathrm{mg} / \mathrm{mL}) \\
\end{array}$} \\
\hline & & $\overline{\mathrm{OE}}$ & & \multirow[t]{2}{*}{$\begin{array}{l}\text { Penicilina } \\
(1 \mathrm{UI} / \mathrm{mL}) \\
\end{array}$} & \multirow[t]{2}{*}{$\begin{array}{c}\text { Nistatina } \\
(20 \mathrm{UI} / \mathrm{mL}) \\
\end{array}$} & \\
\hline & $10 \mathrm{mg}$ & $25 \mathrm{mg}$ & $50 \mathrm{mg}$ & & & \\
\hline Staphylococcus aureus & $10,60 \pm 0,57^{\mathrm{a}}$ & $10,30 \pm 0,57^{\mathrm{a}, \mathrm{b}}$ & $10,16 \pm 0,28^{b}$ & $15,33 \pm 0,57^{\mathrm{c}}$ & NT & 2,5 \\
\hline \multicolumn{7}{|l|}{ ATCC 25923} \\
\hline Streptococcus mutans & - & - & - & $9,50 \pm 0,57^{\mathrm{d}}$ & NT & NT \\
\hline \multicolumn{7}{|l|}{ ATCC 25175} \\
\hline Pseudomonas aeruginosa & - & - & - & $8,66 \pm 0,57^{\mathrm{e}}$ & NT & NT \\
\hline \multicolumn{7}{|l|}{ ATCC 90271} \\
\hline Escherichia coli & - & - & - & $9,26 \pm 0,25^{f}$ & NT & NT \\
\hline \multicolumn{7}{|l|}{ ATCC 10530} \\
\hline Candida albicans & - & - & - & NT & $8,75 \pm 0,26^{\mathrm{g}}$ & NT \\
\hline ATCC 10231 & & & & & & \\
\hline
\end{tabular}

OE: óleo essencial; NT: não testado. Os espaços em branco (-) indicam ausência de halo de inibição. ${ }^{\#}$ Os resultados são expressos como média \pm desvio-padrão $(n=3)$. Significância estatística foi calculada pela ANOVA, seguido pelo teste de Tukey, $\mathrm{p}<0,05$ foi utilizado como nível de significância. Médias seguidas pela mesma letra não diferem entre si, pelo teste de Tukey $(\mathrm{p}<0,05)$. 
Tabela 2. Determinação da $\mathrm{CI}_{50}(\mu \mathrm{g} / \mathrm{mL})$ do óleo essencial e frações obtidas das sementes de Pterodon emarginatus, frente às formas promastigotas de L. amazonensis e L. chagasi ${ }^{\#}$.

\begin{tabular}{lcc}
\hline Amostras & $\mathrm{CI}_{50}(\mu \mathrm{g} / \mathrm{mL})$ L. amazonensis & $\mathrm{CI}_{50}(\mu \mathrm{g} / \mathrm{mL})$ L. chagasi \\
\hline Óleo Essencial & $>100$ & $>100$ \\
Fração Hexânica & $50,06 \pm 1,16^{\mathrm{a}}$ & $>100$ \\
Fração Butanólica & $46,65 \pm 3,04^{\mathrm{b}}$ & $>100$
\end{tabular}
Anfotericina B
$0,90 \pm 0,05^{\mathrm{c}}$
$1,90 \pm 0,12^{\mathrm{d}}$

\#Os resultados são expressos como média \pm desvio-padrão $(n=3)$. Significância estatística foi calculada pela ANOVA, seguido pelo teste de Tukey, $\mathrm{p}<0,05$ foi utilizado como nível de significância. Médias seguidas pela mesma letra não diferem entre si, pelo teste de Tukey $(\mathrm{p}<0,05)$.

promastigotas de L. amazonensis foram cultivadas em meio Warren (BD, Estados Unidos da América) e as formas promastigotas de L. chagasi foram mantidas em meio 199 (GIBCO, Bahamas), ambos suplementados com $10 \%$ de soro fetal bovino. O screening foi realizado em placas de 96 poços, mantidas a temperatura de $24^{\circ} \mathrm{C}$ em estufa. Foram adicionados a cada poço da placa $100 \mu \mathrm{L}$ da suspensão padronizada (L. amazonensis 2 milhões de células/mL e L. chagasi - 3 milhões de célula/mL) e as amostras (OE e frações obtidos de $P$. emarginatus nas concentrações de 6,25 - $100 \mu \mathrm{g} / \mathrm{mL}$ ) dissolvidas com solução de dimetilsulfóxido $0,08 \%$. O procedimento foi realizado em triplicata. A viabilidade celular de ambas as formas promastigotas foram determinadas, após 24 horas, empregando o método do difenil tetrazólio (MTT) (Mosmann, 1983). Os resultados foram expressos através da determinação da concentração inibitória $\left(\mathrm{CI}_{50}\right)$ de cada amostra-teste, que representa a concentração molecular que inibe $50 \%$ do crescimento celular. Anfotericina B foi utilizada como droga de referência.

\section{Análise estatística}

Os resultados foram expressos como média \pm desvio padrão $(n=3)$. Análises de variância ANOVA seguida pelo teste de Tukey foram utilizadas e o valor de $\mathrm{p}$ menor que 0,05 foram utilizados como nível de significância.

\section{RESULTADOS E DISCUSSÃO}

A Tabela 1 apresenta os valores das zonas de inibição do crescimento bacteriano $(\mathrm{mm})$ observados para o OE obtido das sementes de $P$. emarginatus, frente aos microorganismos $S$. aureus ATCC 25923, S. mutans ATCC 25175, P. aeruginosa ATCC 90271, E. coli ATCC 10530 e C. albicans ATCC 10231. O
OE obtido das sementes de $P$. emarginatus causou inibição no crescimento de $S$. aureus (CIM $=2,5$ $\mathrm{mg} / \mathrm{mL}$ ), demonstrando atividade bactericida. Este microorganismo é responsável por quadros de piodermites, abscessos, pneumonia, meningite e septicemia (Jawetz et al., 2000). Esta atividade pode ser decorrente da presença dos constituintes transcariofileno e $\alpha$-humuleno, uma vez que já foi relatada tal atividade para estes constituintes (Pichette et al., 2006; Sonboli et al., 2006). O OE não apresentou atividade antimicrobiana contra $S$. mutans, $P$. aeruginosa, E. coli e C. albicans.

O entusiasmo em relação ao uso de plantas medicinais e seus extratos na assistência à saúde podem ser entendidos pela sua aceitabilidade, derivada da inserção cultural e pela atual disponibilidade desses recursos, ao contrário do que ocorre com outros medicamentos, que na sua maioria são dependentes de matéria-prima e tecnologias externas (Schenkel et al., 1985; Simões et al., 1986). Segundo Verdi et al. (2005), o uso de plantas para o tratamento de diferentes patologias, incluindo as infecciosas, tem sido extensivamente utilizado pelas pessoas, mesmo estas não tendo comprovação científica. Além disso, a grande incidência de infecções, principalmente em indivíduos imunocomprometidos, justifica a realização de estudos científicos para a descoberta de novos compostos terapêuticos oriundos de material vegetal e/ou por via biotecnológica (Carriconde et al., 1996).

A Tabela 2 apresenta os valores da concentração inibitória $\left(\mathrm{CI}_{50}\right)$ de cada amostra-teste frente às formas promastigotas de L. amazonensis e L. chagasi, associadas a todas as formas clínicas das leishmanioses e à leishmaniose visceral, respectivamente (Grimaldi \& Tesh, 1993). As FH $\left(\mathrm{CI}_{50}=50,06 \mu \mathrm{g} / \mathrm{mL}\right)$ e $\mathrm{FB}\left(\mathrm{CI}_{50}\right.$ $=46,65 \mu \mathrm{g} / \mathrm{mL})$ apresentaram atividade leishmanicida frente às formas promastigotas de L. amazonensis, sendo que as mesmas não apresentaram atividade frente 
a L. chagasi. O OE, assim como a FM não apresentaram atividade frente às formas promastigotas de ambas as espécies de Leishmania utilizadas. Os resultados apresentados neste estudo sugerem uma atividade leishmanicida da FH e FB quando comparados com a anfotericina $\mathrm{B}\left(\mathrm{CI}_{50}=0,91 \mu \mathrm{g} / \mathrm{mL}\right)$, utilizado como fármaco de referência, o qual apresentou uma potente atividade leishmanicida confirmando sua aplicação na prática clínica.

Recentes estudos demonstraram a eficácia de algumas plantas medicinais no tratamento de lesões ulceradas da leishmaniose tegumentar e também em modelos in vitro (Akendengue et al., 1999; Bezerra et al., 2006; Paula-Junior et al., 2006). Neste contexto, os produtos naturais têm um grande potencial na pesquisa por novos e seletivos agentes para o tratamento de importantes doenças causadas por protozoários (Phillipson \& Wright, 1991a,b).

Compostos químicos isolados de diversos extratos vegetais demonstraram comprovada atividade leishmanicida in vitro sobre formas promastigotas e/ ou amastigotas de Leishmania. Dentre eles, podem ser citados os terpenóides (Yang \& Liew, 1992; Camacho et al., 2000), aminoglicosteróides e aminosteróides (Kam et al., 1997), naftoquinonas (Fournet et al., 1992a; Kayser et al., 2000), chalconas (Chen et al., 1993), glicosídios iridóides (Mittal et al., 1998); flavonóides (Araújo et al., 1998), neolignanas (Barata et al., 2000) e os alcalóides (Queiroz et al., 1996; Akendengue et al., 1999; Rodríguez et al., 2008). Diversos autores identificaram alguns destes constituintes nas sementes de P. emarginatus (Braz-Filho \& Gottlieb, 1971; Fascio et al., 1976; Torrenegra et al., 1989; Marques et al., 1998; Arriaga et al., 2000), por esta razão, acredita-se que a atividade leishmanicida demonstrada pelas FH e FB possa estar relacionada à presença destes compostos.

Os resultados do presente trabalho demonstraram que o OE obtido das sementes de $P$. emarginatus inibiu o crescimento de $S$. aureus ATCC 25923 e as FH e FB apresentaram expressiva atividade in vitro para L. amazonensis. Esses resultados indicam que moléculas bioativas nas sementes de $P$. emarginatus podem ser utilizadas como protótipos para o desenvolvimento de novos fármacos e/ou como fonte de matérias-primas farmacêuticas antimicrobiana e leishmanicida.

\section{AGRADECIMENTOS}

Os autores são gratos pelo suporte financeiro fornecido pela Coordenação de Aperfeiçoamento de Pessoal de Nível Superior (CAPES), Brasil. R.C.D e F.G.B são alunos bolsista de mestrado pela CAPES. Os autores agradecem a Prof $^{a}$. Dra ${ }^{\mathrm{a}}$. Fátima Regina Gonçalves Salimena Pires pela identificação do material vegetal.

\section{REFERÊNCIAS}

Agra MF, França PF, Barbosa-Filho JM 2007. Synopsis of the plants known as medicinal and poisonous in Northeast of Brazil. Rev Bras Farmacogn 17: 114-140.

Agra MF, Silva KN, Basílio IJLD, França PF, Barbosa-Filho JM 2008. Survey of medicinal plants used in the region Northeast of Brazil. Rev Bras Farmacogn 18: 472508.

Akendengue B, Ngou-milama E, Laurens A, Hocquemiller $\mathrm{R}$ 1999. Recent advances in the fight against leishmaniasis with natural products. Parasite 6: 3-8.

Araújo CAC, Alegrio LV, Leon LL 1998. Antileishmanial activity of compounds extracted and characterized from Centrolobium sclerophyllum. Phytochemistry 49: 751-754.

Arriaga AMC, Castro MAB, Silveira ER, Braz-Filho R 2000. Further diterpenoids isolated from Pterodon polygalaeflorus. J Braz Chem Soc 11: 187-190.

Barata LES, Santos, LS, Ferri PH, Phillipson JD, Paine A, Croft SL 2000. Anti-leishmanial activity of neolignans from Virola species and synthetic analogues. Phytochemistry 55: 589-595.

Bezerra JL, Costa GC, Lopes TC, Carvalho ICDS, Patrício FJ, Sousa SM, Amaral FMM, Rebelo JMM, Guerra RNM, Ribeiro MNS, Nascimento FRF 2006. Avaliação da atividade leishmanicida in vitro de plantas medicinais. Rev Bras Farmacogn 16 (Supl.): 631-637.

Braga FG, Coimbra ES, Matos MO, Carmo AML, Cancio MD, Da Silva AD 2007. Synthesis and biological evaluation of some 6-substituted purines Eur J Med Chem 42: 530-537.

Braz Filho R, Gottlieb OR, Assumpção RMV 1971. The isoflavones of Pterodon pubescens. Phytochemistry 10: $2835-2836$

Caetano N, Saraiva A, Pereira R, Carvalho D, Pimentel MCB, Maia MBS 2002. Determinação de atividades antimicrobiana de extratos de plantas de uso popular como antiiflamatório. Rev Bras Farmacogn 12 (Supl.): 132-135.

Camacho MR, Phillipson JD, Croft LS, Kirby GC, Warhurst DC, Solis PN 2000. Terpenoids from Guarea rhophalocarpa. Phytochemistry 56: 203-210.

Candan F, Unlu M, Tepe B, Daferera D, Polissiou M, Sökmen A, Akpulat A 2003. Antioxidant and antimicrobial activity of essential oil and methanol extracts of Achillea millefolium subsp. millefolium Afan. (Asteraceae). J Ethnopharmacol 87: 215-220.

Carriconde C, Mores D, Von Fritschen M, Cardozo-Junior EL 1996. Plantas medicinais e alimentícias. Olinda: Centro Nordestino de Medicina Popular; Universidade Federal Rural de Pernambuco. v.1, p.45-47.

Chen M, Christensen SB, Blom J, Lemmich E, Nadelmann L, Fich K, Theander TG, Kharazmi A 1993. Licochalcone A, a novel antiparasitic agent with potent activity against human pathogenic protozoan species of Leishmania. Antimicrob Agents Chemother 37: 25502556.

Corrêa MFP, Melo GO, Costa SS 2008. Substâncias de origem vegetal potencialmente úteis na terapia da Asma. Rev Bras Farmacogn 18 (Supl.): 785-797.

Coutinho HDM, Costa JGM, Siqueira-Júnior JP, Lima EO 2008. In vitro anti-staphylococcal activity of Hyptis martiusii Benth against methicillin-resistant Staphylococcus aureus-MRSA strains. Rev Bras Farmacogn 18 (Supl.): 670-675.

Croft SL, Yardley V 2002. Chemotherapy of leismaniasis. Curr 
Pharm Design 8: 319-342.

Dos Santos Filho D, Vichnewski W, Baker PM, Gilbert B 1972. Prophylaxis of Schistosomiaris diterpenes from Pterodon pubenscens. An Acad Bras Cienc 44: 45-49.

Fascio M, Mors WB, Gilbert B, Mahajan JR, Monteiro MB, Dos Santos Filho D, Vichenewski W 1976. Diterpenoid Furans from Pterodon species. Phytochemistry 15: 201-203.

Fournet A, Barrios AA, Munõz V, Hocquemiller CA 1992a. Effects of natural naphtoquinones in $\mathrm{Balb} / \mathrm{c}$ mice infected with Leishmania amazonensis and $L$. venezuelensis. Ann Trop Med Parasit 43: 219-222.

Grevelink SA, Lerner EA 1996. Leishmaniasis. J Am Acad Dermatol 34: 257-272.

Grimaldi GJr, Tesh RB 1993. Leishmaniasis of New World: Current concepts and implication for future research. Clin Microbiol Rev 6: 230-250.

Herwaldt BL 1999. Leishmaniais. Lancet 354: 1191-1199.

Jawetz E, Melnick JL, Adelberg, EA, Brooks GF, Butel JS, Ornston LN 2000. Microbiologia médica, 21th edn. São Paulo: Editora Guanabara Koogan.

Kam TS, Sim KM, Koyana T, Toyoshima M, Hayash M, Komiyama K 1997. Citotoxic and Leishmanicidal aminoglycosteroids and aminosteroids from Holarrhena curtisii. J Nat Prod 61: 1332-1336.

Kayser O, Kiderlen AF, Laatsch H, Croft SL 2000. In vitro leishmanicidal activity of monomeric and dimeric naphthoquinones. Acta Tropica 77: 307-314.

Leite de Almeida ME, Gottlieb OR 1975. The chemistry of Brazilian Leguminosae, further isoflavones from Pterodon apparicia. Phytochemistry 14: 12, 2716.

Lorenzi H, Matos FJA 2002. Plantas medicinais no Brasil: nativas e exóticas. São Paulo: Instituto Plantarum.

Marques DD, Machado MIL, Carvalho MG, Meleira LAC, Braz-Filho R 1998. Isoflavonoids and triterpenoids isolated from Pterodon polygalaeflorus. J Braz Chem Soc 9: 295-301.

Matthias LA, Emily A 1993. Tapping and Amazonian plethora: four medicinal plants of Marajó Island, Pará-Brazil. $J$ Ethnopharmacol 40: 53-75.

Ministério da Saúde 2000. Manual de Controle da Leishmaniose Tegumentar Americana. Brasília: Centro Nacional de Epidemiologia, FUNASA, MS.

Mislivec PB, Beuchat LR, Causin MA 1992. Yeast and molds. In Compendium of methods for the microbiological examination offood, 3rd edn. American Public Health Association: Washington, 239-249.

Mittal N, Gupta N, Saksena S, Goyal N, Roy U, Rastogi AK 1998. Protective effect of picrolive from Pichrorhiza kurroa against Leishmania donovani infections in Mesocricetus auratus. Life Sci 63: 1823-1834.

Mosmann T 1983. Rapid colorimetric assay for cellular growth and survival: application to proliferation and cytotoxicity assays. J Immunol Methods 16: 55-63.

Nascimento GGF, Locatelli J, Freitas PC, Silva GL 2000. Antibacterial activity of plant extracts and phytochemicals on antibiotic-resistant bacteria. Braz J Microbiol 31: 247-256.

Oliveira FP, Lima EO, Siqueira Júnior JP, Souza EL, Santos BHC, Barreto HM 2006a. Effectiveness of Lippia sidoides Cham. (Verbenaceae) essential oil in inhibiting the growth of Staphylococcus aureus strains isolated from clinical material. Rev Bras Farmacogn 16: 510-516.

Oliveira RAG, Lima EO, Vieira WL, Freire KRL, Trajano VN, Lima IO, Souza EL, Toledo MS, Silva-Filho RN 2006b. Estudo da interferência de óleos essenciais sobre a atividade de alguns antibióticos usados na clínica. Rev Bras Farmacogn 16: 77-82.

Paula-Junior W, Rocha FH, Donatti L, Fadel-Picheth CMT, Weffort-Santos AM 2006. Leishmanicidal, antibacterial, and antioxidant activities of Caryocar brasiliense Cambess leaves hydroethanolic extract. Rev Bras Farmacogn 16 (Supl.): 625-630.

Phillipson JD, Wright CW 1991a. Medicinal plants in tropical medicine: Medicinal plants against protozoal diseases. Trans Royal Soc Trop Med Hyg 85: 18-21.

Phillipson JD, Wright CW 1991b. Antiprotozoal agents from plant sources. Planta Med 57: 53-59.

Pichette A, Larouche PL, Lebrun M, Legault J 2006. Composition and antibacterial activity of Abies balsame an essential oil. Phytother Res 20: 371-373.

Queiroz EF, Roblot F, Cave A, Paulo MD, Fournet A 1996. Pessoine and spinosine, two catecholic berbines from Annona spinescens. J Nat Prod 59: 438-440.

Rath S, Trivelin LA, Imbrunito TR, Tomazela DM, Jesus MN, Marzal PC, Andrade Júnior HF, Tempone AG 2003. Antimoniais empregados no tratamento da leishmaniose: estado da arte. Quim Nova 26: 550-553.

Rocha LG, Almeida JRGS, Macedo RO, Barbosa-Filho JM 2005. A review of natural products with antileishmanial activity. Phytomedicine 12: 514-535.

Rodríguez AM, Camargo JR, García FJB 2008. Actividad in vitro de la mezcla de alcaloides de Ervatamia coronaria (Jacq) Staff. Apocynaceae sobre amastigotes de Leishmania braziliensis. Rev Bras Farmacogn 18: 350-355.

Sakagami Y, Kajamura K 2006. Bactericidal activities of desinfectants against vancomycin-resistant Enterococci. J Hosp Infect 56: 140-144.

Schenkel EP, Simões CMO, Mengue SS, Mentz LA, Irgang BE, Stehmann JR 1985. O espaço das plantas medicinais e suas formas derivadas na medicina científica. Caderno de Farmácia 1: 65-72.

Shiomori T, Miyamoto H, Makashima K, Yoshida M, Fujiyoshi T, Udaka T, Inaba T, Hiraki N 2002. Evaluation of bedmaking-related airborne and surface methicillinresistant Staphylococcus aureus contamination. J Hosp Infect 50: 30-35.

Simões CMO, Mentz LA, Schenkel EP, Irgang BE, Stehmann JR 1986. Plantas da medicina popular no Rio Grande do Sul. Porto Alegre: Editora da Universidade UFRGS.

Sonboli A, Babakhani B, Mehrabian AR 2006. Antimicrobial activity of six constituents of essential oil from Salvia. J Biosciences 61: 160-164.

Sousa FCF, Melo CTV, Citó MCO, Félix FHC, Vasconcelos SMM, Fonteles MMF, Barbosa-Filho JM, Viana GSB 2008. Plantas medicinais e seus constituintes bioativos: Uma revisão da bioatividade e potenciais benefícios nos distúrbios da ansiedade em modelos animais. Rev Bras Farmacogn 18: 642-654.

Swanson KMJ, Busta FF, Peterson EH, Johanson MG 1992. Colony count methods. In Compendium of methods for the microbiological examination of food, 3rd edn., 
Vanderzant, C.; Splittstoesser, D.F. (eds). American Public Health Association: Washington, 239-249.

Torrenegra R, Bauereib P, Achenbach H 1989. Homoormosanine-type alkaloids from Bowdichia virgiloides. Phytochemistry 28: 2219-2221.

Verdi LG, Brighente IMC, Pizzolatti MG 2005. O gênero Baccharis (Asteraceae): Aspectos químicos, econômicos e biológicos. Quim Nova 28: 85-94.

Yang DM, Liew FY 1992. Effects of quinghaosu (artemisin) and its derivates on experimental cutaneos leismaniasis. Parasitol 6: 7-11. 